\title{
Quiste leptomeníngeo orbitario desarrollado tras sufrir un trauma en la propia adultez
}

\section{Orbital Intradiploic Leptomeningeal Cyst Development after Adult Trauma}

\author{
C Lesly Solís Alfonso ${ }^{10}$ \\ ${ }^{1}$ Instituto Cubano de Oftalmología Ramón Pando Ferrer, Marianao, La \\ Habana, Cuba \\ Rev Argent Radiol 2021;85:49-51.
}

Estimados editores,

El quiste leptomeníngeo, también llamado fractura craneal evolutiva, creciente, o expansiva, meningocele traumático, pseudomeningocele, o absorción ósea postraumática, es una rara complicación de las fracturas craneales, siendo su incidencia inferior al $1 \%$. Ocurre de forma casi exclusiva en niños menores de tres años, pero se han reportado algunos pocos en adultos, que se atribuyen a fracturas craneales sufridas en la infancia. ${ }^{1-3}$

Se presenta el caso de una paciente femenina de 51 años, que es remitida a consulta de Oculoplastia por notar desde hace aproximadamente cuatro años una proptosis derecha no dolorosa y de progresión lenta, la cual apareció tras recibir un trauma contuso a este mismo nivel. Al examen físico, se corroboró la proptosis y se constató edema palpebral, por lo que se realizó tomografía computada (TC) simple, que reveló una lesión osteolítica en el techo de la órbita derecha, con densidades medias internas entre 12 y 30 Unidades Hounsfield, la cual expandía el hueso frontal comprometido, adelgazando y rompiendo parcialmente la tabla externa, mientras que en la interna se descubrió una solución de continuidad, de $\pm 8 \mathrm{~mm}$ en sentido latero-lateral. Dicha imagen ocupante de espacio ocasionaba desplazamiento antero-inferior del globo ocular, e inferior del complejo muscular recto superior-elevador del párpado superior y del complejo nervio óptico-vaina. Además, se comprobó independiente del seno frontal, llegando a medir $\pm 29 \times 24 \times 13 \mathrm{~mm}$ en sentido rostro-caudal, latero-

received January 28, 2020 accepted August 25, 2020
Address for correspondence C. Lesly Solís Alfonso, PhD, Profesor Titular, Calle 411, e/ 192 y 194, Santiago de las Vegas, Boyeros, La Habana, 10800, Cuba (e-mail: leslysa@infomed.sld.cu).

lateral y dorso-ventral respectivamente (-Fig. 1). Seguidamente, se hizo una resonancia magnética (RM) donde la lesión del techo orbitario se comportó prácticamente isointensa, con respecto al LCR, tanto en las secuencias potenciadas en $\mathrm{T} 1$ como en las ponderadas en T2; y se confirmó el resto de los hallazgos tomográficos previamente descritos (-Fig. 2). Posteriormente, se efectuó un scan radiográfico óseo sin demostrarse lesiones osteolíticas adicionales, así como ultrasonido de cuello, mamas y abdomen, y radiografía de tórax, sin evidenciarse una posible lesión primaria. Teniendo en cuenta lo expuesto, se planteó el diagnóstico de fractura orbitaria evolutiva, con herniación de meninges y de pequeña porción encefálica, asociado a extensión intradiploica del componente quístico. La paciente se sometió a neurocirugía y se le realizó reparación quirúrgica del defecto dural, mediante el empleo de material autólogo (fascia lata), lográndose una evolución satisfactoria con remisión de la proptosis (- Fig. 3).

Para la formación del quiste leptomeníngeo es imprescindible la presencia de una fractura craneal amplia, que generalmente se acompaña de lesión cerebral subyacente, asociado a desgarro de la duramadre, lo que condiciona el desvío del LCR a esa zona de menor presión. Al mismo tiempo, las pulsaciones del LCR ocasionan diástasis de la fractura, con la consiguiente erosión de los bordes óseos, y herniación de las meninges y/o del encéfalo a través del defecto dural. ${ }^{2,4}$ En ese sentido, algunos autores han sugerido su asociación con la osteopenia de la prematuridad, lo que
DOI https://doi.org/ $10.1055 / \mathrm{s}-0040-1721338$. ISSN 1852-9992.

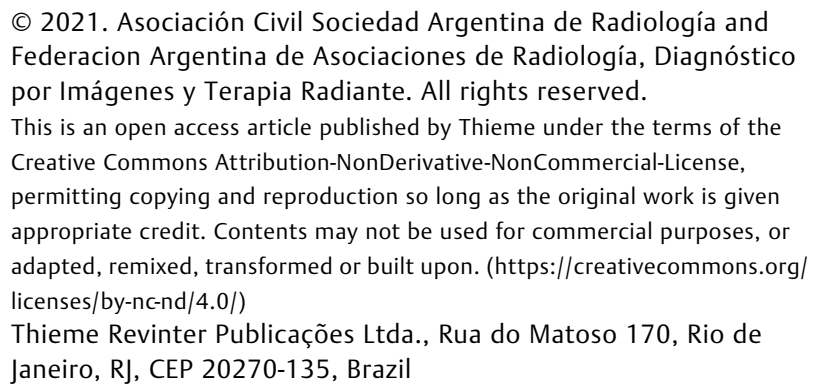



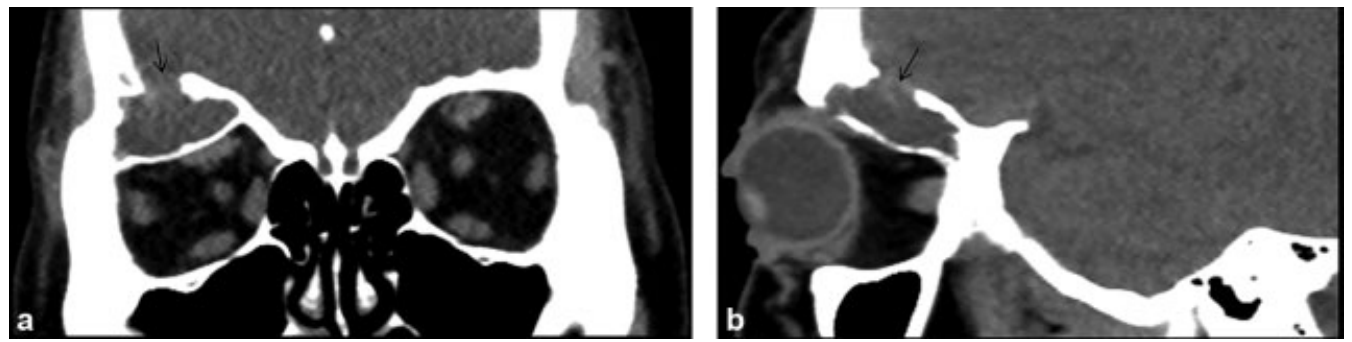

Fig. 1 (a y b) TC simple de cráneo: reconstrucción coronal y sagital, donde se evidencia el defecto óseo en techo de órbita derecha (flecha) y la extensión intradiploica del componente quístico.
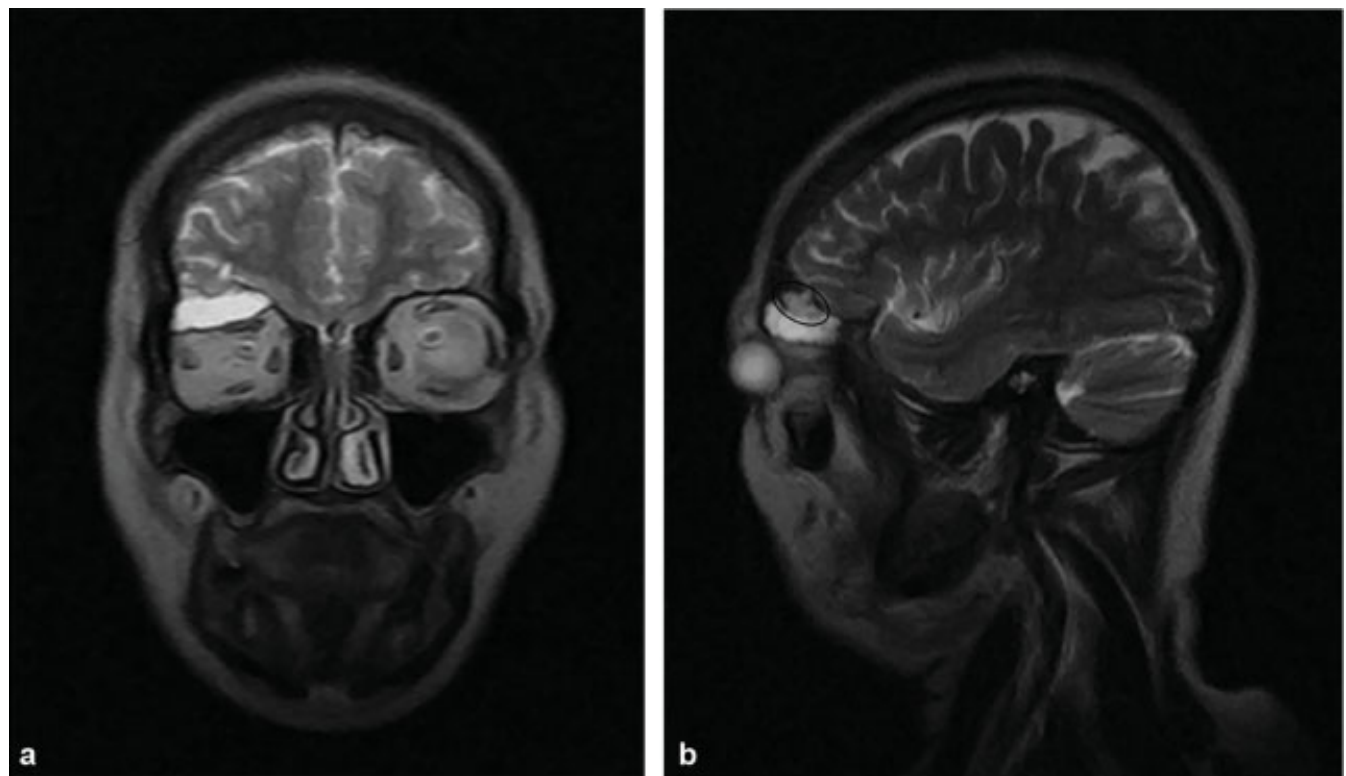

Fig. 2 (a y b) RM de cráneo: secuencias coronal y sagital potenciadas en T2, donde se confirma la fractura evolutiva del techo orbitario derecho, la extensión intradiploica del componente quístico y se demuestra leve protrusión del tejido cerebral a través del defecto dural (círculo).
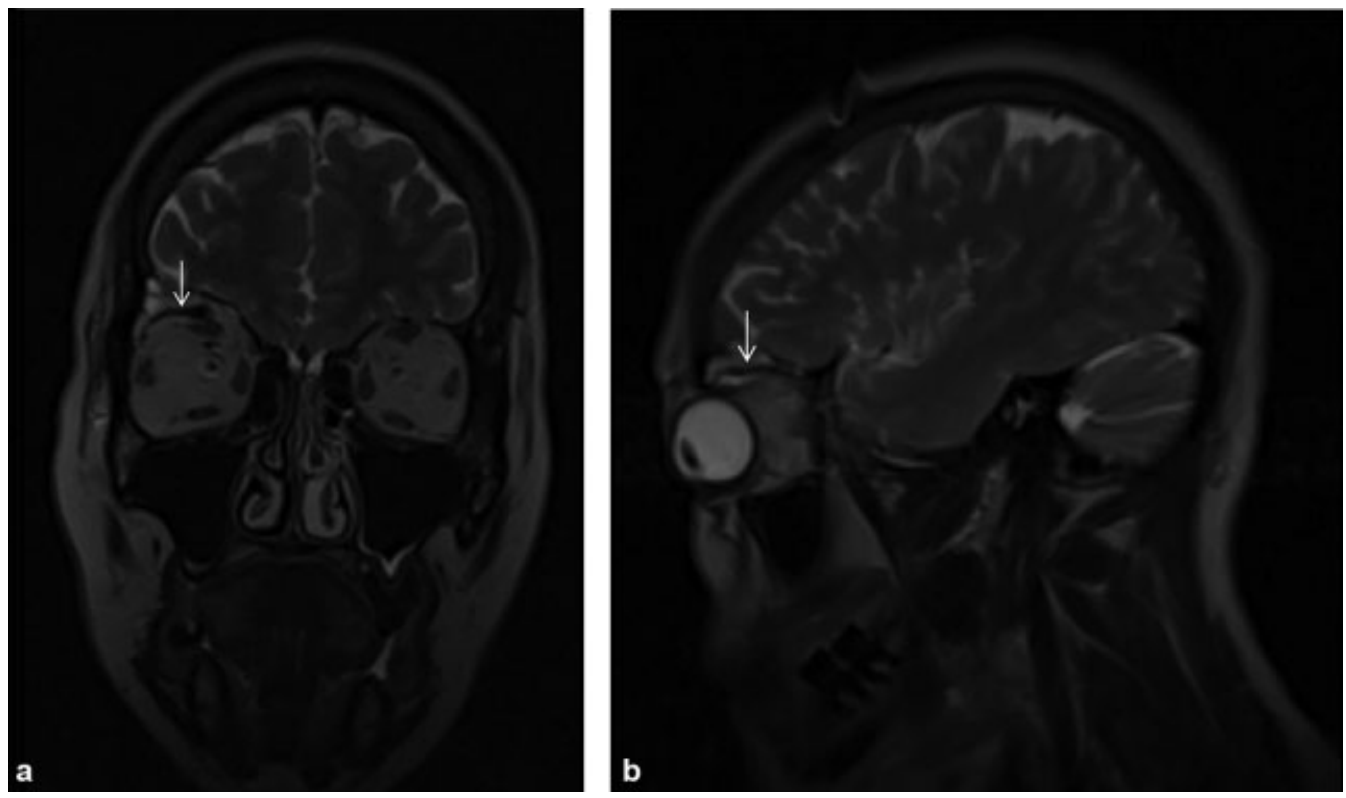

Fig. 3 ( a y b) RM evolutiva al mes de la cirugía: secuencias coronal y sagital ponderadas en T2, donde se define el segmento de fascia lata con que se reparó el defecto dural (flecha). 
hace suponer que la osteoporosis postmenopáusica también podría favorecer la evolución tórpida de una fractura craneal en la adultez. ${ }^{2}$

Por otra parte, suele manifestarse como una tumoración circunscrita, progresiva y persistente en cuero cabelludo, o con síntomas neurológicos, siendo los más comunes las convulsiones y la hemiparesia.,6 Esporádicamente, también se han reportado inflamación de párpados $\mathrm{y}$ proptosis. $^{6,7}$

La localización más frecuente es la región parietal o fronto-parietal, aunque se han descrito otras como el hueso occipital, temporal, incluso la órbita. ${ }^{1-5,7,8}$ Además, se ha planteado que ocasionalmente el crecimiento quístico podría ser intradiploico. ${ }^{4,8,9}$

Lo más raro de este caso radica en reunir todas las características inusuales que aquí se han detallado. En particular, destaca el hecho de sufrir el trauma desencadenante en la propia adultez.

La TC continúa siendo la prueba de elección para la valoración de los pacientes que han sufrido un trauma craneal, representando la clave diagnóstica de esa infrecuente complicación. En la actualidad, su empleo sistemático permite la detección temprana del quiste leptomeníngeo, lo que mejora el pronóstico de esa afección., 5,

El uso complementario de la RM pretende definir mejor el contenido del quiste y el grado de lesión parenquimatosa, lo que facilita el diagnóstico diferencial, el cual incluye otras lesiones óseas tales como: las metástasis, el mieloma, el granuloma eosinófilo y el quiste aracnoideo intradiploico. En consecuencia, se optimiza la planificación del tratamiento. ${ }^{2,3,10}$

Pese a que se han reportado casos de estabilización espontánea de fracturas craneales evolutivas, sigue siendo la cirugía inmediata lo más recomendado, con el propósito de prevenir complicaciones. ${ }^{3}$ Este proceder quirúrgico suele ser curativo y consiste en el cierre hermético de la duramadre, el retiro del tejido gliótico y la corrección del defecto óseo. ${ }^{2,4-6}$

Por lo tanto, frente a todo trauma craneal, independientemente de la edad del paciente, siempre se deberá considerar al quiste leptomeníngeo como una posible complicación a largo plazo.
Confidencialidad de los datos

La autora declara que ha seguido los protocolos de su centro de trabajo sobre la publicación de datos de pacientes, y que todos los pacientes incluidos en el estudio han recibido información suficiente $\mathrm{y}$ han dado su consentimiento informado por escrito para participar en dicho estudio.

\section{Conflicto de intereses}

La autora del trabajo declara no tener ningún conflicto de intereses.

\section{Bibliografía}

1 Castro GFJ, Asensio CJF, Adeva BMT, García IP. Quiste leptomeníngeo. Hallazgos en TC y RM. Rev Chil Radiol. 2004;10 (03):129-131

2 San Martín-García I, Aguilera-Albesa S, Zazpe-Cenoz I, Yoldi-Petri ME. Fractura craneal evolutiva postraumática recidivada. Rev Neurol. 2015;60(08):351-354

3 Meier JD, Dublin AB, Strong EB. Leptomeningeal cyst of the orbital roof in an adult: case report and literature review. Skull Base. 2009;19(03):231-235

4 Reyes Graterol EO, Chacín González JP, Zumztein López DJ, García Oduber SM. Fractura evolutiva; colocación de sistema derivativo cisto-peritoneal y corrección diferida. A propósito de un caso. Rev Chil Neurocirugía. 2015;41(02):120-123

5 Gelabert-González M, García-Pravos A, Fernández-Villa JM, Cutrín-Prieto JM, Pérez-Muñuzuri A. Quiste leptomeníngeo postraumático de larga evolución. Neurocirugia (Astur). 2000; 11(03):224-230

6 Diyora B, Nayak N, Kamble H, Kukreja S, Gupte G, Sharma A. Surgical treatment and results in growing skull fracture. Neurol India. 2011;59(03):424-428

7 Satyarthee GD, Kumar A. Posttraumatic giant fronto-orbito encephalocele causing cosmetic disfiguring forehead swelling with proptosis: Management. J Pediatr Neurosci. 2016;11(04):341-343

8 Escudero R, Pérez I, Roldán F, Canabal A. Fractura craneal evolutiva. Extensión intradiploica occipital del quiste leptomeníngeo. Radiologia (Madr). 2001;43(06):287-289

9 Karki KT, Chaudhary PK, Munakomi S, Bhattarai B, Cherian I. Post traumatic intradiploic leptomeningeal cyst in a 2 year old child. J Pediatr Neurol Med. 2016;1(01):1-3

10 de Araújo Neto FB, Valois VM, Dias MV, Furlan S, Fugita DYA. Posttraumatic intraosseous leptomeningeal cyst. Radiol Bras. 2018;51 (02):126-128 